\title{
"Plasmocitoma-like" gástrico en un paciente trasplantado cardíaco. Revisión de algunos conceptos etiopatogénicos y terapéuticos
}

\author{
J. A. Rivera Bautista ${ }^{1}$, M. D. Chaparro Cabezas ${ }^{1}$, C. Salas Antón ${ }^{2}$, J. Segovia Cubero ${ }^{3}$, \\ D. Melero Montes ${ }^{1}$, A. Colás Vicente ${ }^{1}$
}

\section{Resumen}

El Plasmocitoma-like es una entidad poco frecuente englobada dentro de los trastornos linfoproliferativos postransplante (TLPT). La inmunosupesión y la infección con virus de Epstein-Barr (VEB) juegan un papel primordial en su desarrollo. $\mathrm{Su}$ diagnóstico preoperatorio es difícil si no se aplican estudios inmunohistoquímicos sobre las biopsias, y el tratamiento adecuado debe ser quirúrgico y/o radioterápico, asociado a la reducción de la dosis de inmunosupresores. El seguimiento de estos enfermos debe ser estrecho ya que puede ocurrir la recurrencia del tumor, así como la aparición de rechazo del trasplante por la disminución de los inmunosupresores.

Presentamos un paciente trasplantado cardíaco que desarrolló un TLPT tipo "plasmocitoma-like" en el estómago siete años después del trasplante, comentamos las teorías etiopatogénicas, los criterios diagnósticos y sus posibilidades de tratamiento.

Palabras clave: Plasmocitoma-like. Trastorno linfoproliferativo postransplante (TLPT). Virus de Epstein-Barr (VEB). Inmunosupresión.

Oncología, 2006; 29 (7):307-312

\section{Summary}

Plasmacytoma-like tumour is an infrequent entity observed among the post-transplant lymphoproliferative disorders (PTLD). Immunosuppression and Epstein.Barr virus (EBV) infection play an important role in the development of the process. The preoperative diagnosis is difficult, but it can be made by means of immunohistochemical studies of the biopsies. The adequate treatment consists of surgery, radiotherapy and reduction of the administered immunosuppressor drugs. The patients need a close follow up because the resulting immusuppression facilitates the recurrence of the tumour or the rejection of the transplanted organ.

We present the case of a patient with a heart transplant who developed a gastric plasmacytomalike PTLD seven years after transplantation. We discuss the etiopathogenic theories, the diagnostic criteria and the treatment options

Key words: Plamacytoma-like neoplam. Posttransplant lymphoproliferative disorder (PTLD). Epstein-Barr virus (EBV). Immunosuppression.

\footnotetext{
${ }^{1}$ Servicio de Cirugía General y del Aparato Digestivo

${ }^{2}$ Servicio de Anatomía Patológica

${ }^{3}$ Servicio de Cardiología, Unidad de Trasplante Cardíaco

Hospital Universitario Puerta de Hierro

Madrid (España)
}

Recibido: 20.04 .06

Revisado: 28.04 .06

Aceptado: 05.05.06 


\section{Introducción}

Los pacientes que han sido sometidos a un trasplante de órgano sólido tienen un mayor riesgo que la población general de desarrollar trastornos neoplásicos malignos. Tras los tumores cutáneos los trastornos linfoproliferativos postransplante (TLPT) son las neoplasias más frecuentes padecidas por estos pacientes, afectan entre un 1-10\% de los trasplantados ${ }^{1,2}$ y son la causa más frecuente de muerte debida al cáncer después de un trasplante, con una mortalidad global superior al $50 \%{ }^{3}$. La inmunosupresión farmacológica, sobre todo con ciclosporina, y determinados virus como el de Epstein-Barr (VEB), están implicados en su patogénesis ${ }^{1,2}$.

\section{Presentación del caso}

Paciente varón de 48 años, transplantado cardíaco desde hacía siete años, que consultó por molestias epigástricas tras la ingesta y plenitud postprandial. Se le realizó una gastroscopia que demostró un gran tumor en antro gástrico que estenosaba la luz, se tomaron muestras para biopsia y determinación de helicobacter pylorii (H. pylorii). El estudio anatomopatológico reveló un proceso linfoproliferativo

El resto de las exploraciones complementarias aportaron los siguientes datos: 9800 leucocitos (72\% neutrófilos) Hb. $9.6 \mathrm{~g} / \mathrm{dl}$, plaquetas $349000 / \mathrm{mm}^{3}$, frotis sanguíneo con anisopoiquilocitosis leve, bioquímica hemática normal. La electroforesis de las proteínas plasmáticas mostraba un patrón de inmunoglobulinas normal, sin pico monoclonal, proteínas en orina negativas, beta 2 microglobulina 1,5. Serie ósea radiológica normal. Biopsia de médula ósea polimorfa sin evidencia de afectación tumoral (células plasmáticas 1-2\%, serie linfoide $10 \%)$. Serología VEB: IgG positiva en el estudio pretrasplante. Gammagrafia con galio negativa. En el EED había un gran tumor gástrico y en la TAC toraco-abdominal un engrosamiento de la pared gástrica sin masas ni adenopatias patológicas a ningún nivel. El test del H. pylorii fue positivo.

Se decidió realizar una laparotomía exploradora, apreciando un tumor de gran tamaño en antro gástrico que infiltraba la cara anterior del páncreas y mesocolon transverso hasta la raíz del mesenterio.

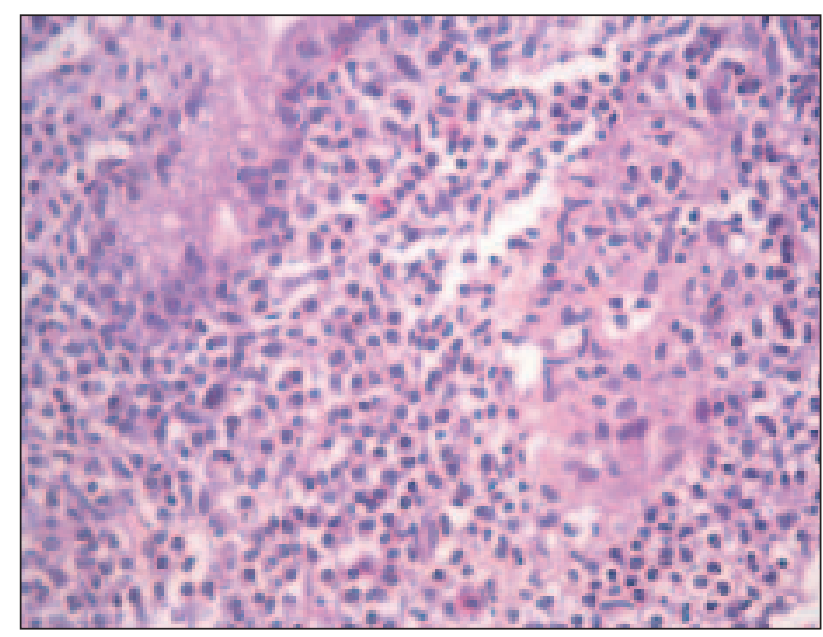

Figura 1. Microfotografía (HE x 125) en la que se aprecia la mucosa gástrica que muestra un denso infiltrado en la lámina propia y desaparición glandular.

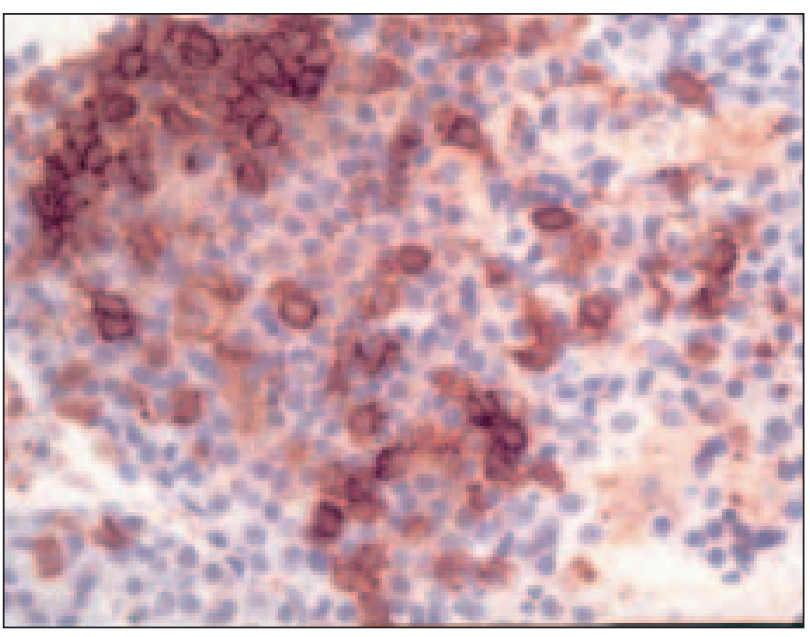

Figura 2. El infiltrado linfoide expresa el anticuerpo monoclonal CD20 tanto en la lámina propia como en el interior de las glándulas.

Se consideró irresecable y se procedió a la realización de una gastroenteroanastomosis paliativa y toma de biopsias.

El estudio histopatológico de las biopsias quirúrgicas mostró un proceso linfoproliferativo que expandía la mucosa con pérdida y desestructuración glandular e infiltraba la capa muscular. La proliferación neoplásica estaba constituida por células de características morfológicas similares a las células plasmáticas, con inclusiones tipo Dutcher y Russell de inmunofenotipo CD20-Kappa (Figs. 1 y 2). La proteína latente de membrana del VEB (LMP-1) era positiva. 
Tras la intervención no se planteó tratamiento radioterápico por considerar que la toxicidad generada en el campo de irradiación era inaceptable. Se administró Ganciclovir IV durante 15 días y se continuó con Aciclovir oral. Recibió tratamiento con Claritromicina y Amoxicilina para tratar la infección por H. pylorii. Se redujo la dosis de Ciclosporina y de Prednisona y se sustituyó la Aziatoprina por Micofenolato Mofetil. En la gastroscopia de control realizada en mayo de ese mismo año, se aprecia una reducción de la masa tumoral. El estudio anatomopatológico de las biopsias informa de reducción importante de las células neoplásicas y la presencia de algún cúmulo linfoide.

Ante estos datos se inicia tratamiento con Interferón a dosis de 1.5 millones de unidades a días alternos. En los siguientes meses, el paciente presenta rechazo agudo-moderado en el injerto que se trata con metilprednisolona en bolus durante 3 días y posteriormente se aumenta la dosis de Ciclosporina, aunque por debajo del rango terapéutico. A los cinco meses del diagnóstico, la gastroscopia, la biopsia y el test de $\mathrm{H}$. pylorii fueron negativos.

Se aumento la dosis de interferón a 3 millones de unidades a días alternos, debido a la buena tolerancia al tratamiento. Posteriormente presentó otro episodio de insuficiencia cardíaca y, ante la sospecha de rechazo agudo, se realizó una biopsia cardíaca que lo confirmó. Se instauró tratamiento con corticoides. En el segundo día del tratamiento se produjo la muerte por arritmia cardíaca. La autopsia mostró la ausencia morfológica del proceso linfoproliferativo gástrico y un rechazo grave del injerto cardíaco.

\section{Discusión}

Los TLPT comprenden un amplio espectro de procesos linfoproliferativos con diferente patogénesis, apariencia histológica y comportamiento clínico. Una aproximación normalizada a la clasificación de estos trastornos es importante para permitir la comparación de los diferentes tratamientos. Según la última clasificación de la OMS se dividen en diversos tipos (Tabla I) ${ }^{4}$. La hiperplasia plasmacítica reactiva es más frecuente en niños y adultos jóvenes durante el primer año postransplante ${ }^{5}$. El TLPT polimórfico es el tipo más común en adultos y puede
TABLA I

Trastornos linfoproliferativos postrasplantes

1. Lesiones precoces:

Hiperplasia plasmacítica reactiva

Mononucleosis infecciosa-like

2. TLPT polimorfos

3. TLPT monomorfos (clasificados conforme a la clasificación de linfomas)

- Neoplasias de células B

Linfoma difuso de célula B grande (inmunoblástico, centroblástico, anaplásico)

Linfoma Burkitt y Burkitt-like

Mieloma de células plasmáticas

Lesiones plasmocitoma-like

- Neoplasias de células T

Linfoma de células $\mathrm{T}$ periféricas (o linfoma $\mathrm{T}$ periférico) no especificado

Otros tipos

4. Linfoma de Hodgkin y Hodgkin like

ocurrir en cualquier momento tras el transplante. Dentro de los TLPT monomórficos, menos comunes y más graves que los dos grupos anteriores y que, a menudo, se ven varios años después del transplante, se incluyen las lesiones plasmocitomalike.

El riesgo de desarrollar un TLPT difiere según el tipo de órgano trasplantado, es menor si es un trasplante renal (1-2,3\%) que si es un trasplante hepático, cardíaco (1-6,3\%), cardíaco-pulmonar $(2,4$ $5,8 \%)$, pulmonar $(4-8,3 \%)$ o, con la mayor incidencia, intestinal $(20 \%)^{1,5,6,7}$. Desde la introducción en el arsenal terapéutico antirrechazo de la ciclosporina se ha objetivado una elevación en el número de casos de TLPT. Asimismo parece que cuando se usa takrolimus (FK506) en vez de ciclosporina se incrementa en más del doble el riesgo de desarrollar uno de estos procesos. La terapéutica de inducción y el tratamiento del rechazo con OKT3 y ATG (gammaglobulina anti-T) también incrementa el riesgo ${ }^{2,3}$.

La inmunidad mediada por linfocitos $\mathrm{T}$ citotóxicos (LTC) gravemente deprimida por la acción de los inmunosupresores incapacita al organismo para eliminar las células transformadas en malignas por la acción de diversos carcinógenos; los mismos inmunosupresores pueden dañar directamente el DNA o incluso potenciar el efecto de otros agentes cancerígenos. La infección por el VEB, relacionada con el $90 \%$ de los casos de TLPT en niños y en el $80 \%$ 
de los casos en adultos ${ }^{8,9}$, favorece la mutación de oncogenes y la transformación maligna de los linfocitos B infectados permitiendo el desarrollo de trastornos linfoproliferativos en pacientes trasplan$\operatorname{tados}^{10,11}$.

El cuadro clínico con el que debutan estos pacientes suele ser bastante inespecífico, principalmente fiebre, linfadenopatía, pérdida de peso, etc. Aproximadamente dos terceras partes de los pacientes tienen enfermedad localizada en un único órgano, siendo los lugares más frecuentemente afectados los ganglios linfáticos, el hígado, los pulmones y el intestino $^{12,2}$. En el tracto gastrointestinal la afectación más frecuente es el ileon terminal y el colon, pudiendo causar obstrucción intestinal, malabsorción y hemorragia digestiva baja ${ }^{13,14}$. Si afecta al estómago los pacientes suelen tener dispepsia, epigastralgia, hemorragia digestiva alta por ulceración tumoral, etc. Cuando se realiza el estudio endoscópico estas lesiones presentan ya un gran tamaño y su aspecto macroscópico es similar al de otras neoplasias gástricas más frecuentes como el adenocarcinoma o el linfoma MALT.

El diagnóstico se basa en el estudio histopatológico de una biopsia del tejido afectado que muestre un proceso linfoproliferativo de células B, positiva para marcadores fenotípicos característicos (CD19, CD20), con población celular oligo o monoclonal basada en la expresión de cadenas $\kappa$ o $\lambda$, y la presencia de VEB en las células ${ }^{16}$. En el caso de un TLPT tipo plasmocitoma-like la simple objetivación de infiltrado de células plasmáticas atípicas en los tejidos no es per se diagnóstica ya que se presenta igualmente en otros procesos como el linfoma tipo MALT, o el pseudotumor inflamatorio con células plasmáticas ${ }^{17}$. El estudio se debe completar con una TAC tóraco-abdominal y un aspirado de médula ósea. En ocasiones puede existir una proteina monoclonal en el suero de estos pacientes, aunque no se ha observado correlación entre la presencia de la misma y la supervivencia global o la recidiva tras tratamiento ${ }^{18}$. Nuestro paciente debutó con epigastralgia y plenitud postprandial, tras realizarse endoscopia digestiva se objetivó la lesión en antro, siendo el diagnóstico histopatológico de trastorno linfoproliferativo y el estudio de extensión negativo. El paciente estaba en tratamiento inmunosupresor con ciclosporina, prednisona y azatioprina; la serología de EVB era positiva antes del trasplante, y no se objetivó en suero ni en orina elevación monoclonal de inmunoglobulina.

Aunque no existe un protocolo establecido para el tratamiento de estos pacientes hay algunos autores que sugieren una determinada línea de actuación, en función de las características del trastorno ${ }^{5}$, lo que pasa por clasificar histológicamente y estadiar los pacientes según los criterios de la $\mathrm{OMS}^{4}$.

El tratamiento optimo de una lesión localizada debe ser quirúrgico, si es resecable. La alternativa es la radioterapia ya sea como tratamiento adyuvante, o primario en lesiones inextirpables o que afecten al $\mathrm{SNC}^{19,5}$.

Disminuir la dosis de inmunosupresores es obligatorio para conseguir la regresión de estos tumores $^{20,21}$. La reducción de la inmunosupresión permite restaurar, al menos parcialmente, la función de los LTC VEB-específicos y la eliminación de los linfocitos B infectados, incluidos los tumorales, pero conlleva un riesgo muy alto de rechazo agudo del injerto, que obliga a una vigilancia muy próxima de la función del órgano trasplantado. La respuesta a la reducción de la inmunosupresión debe ser evaluada usando los métodos diagnósticos adecuados.

Determinar la presencia de antígenos del VEB en el tejido tumoral puede ampliar las posibilidades terapéuticas de estos trastornos con la utilización de agentes antivirales como el aciclovir, valaciclovir y ganciclovir, aunque existen escasas pruebas de que mejoren significativamente el resultado $^{22}$. Otros tratamientos que se han utilizado, solos o combinados entre sí, son los agentes quimioterápicos, para los tipos monomórficos más agresivos de TLPT, el interferón-a que mejora la función de los LTC, el anticuerpo monoclonal antiCD20 Rituximab, que provoca una importante y duradera depleción de linfocitos $\mathrm{B}$ y que ha mostrado unos resultados prometedores en su limitada experiencia, la rapamicina que inhibe el crecimiento de los linfocitos B transformados por el VEB, además de tener propiedades antitumorales, antiangiogénicas e inmunosupresoras, y la transfusión de LTC específicos contra el VEB, que pueden proceder del donante o incluso del propio receptor, si tenía serología positiva para el VEB antes del trasplante ${ }^{23-29}$. Si preoperatoriamente se puede diferenciar el plasmocitoma-like del linfoma tipo MALT 
que se asocia a infección por H. pylorii el tratamiento inicial debe incluir los antibióticos ${ }^{30}$. En nuestro caso no fue posible la resección quirúrgica, pero el tratamiento de la infección por $\mathrm{H}$. pylorii y el tratamiento inmunológico contra el EVB así como la reducción de la dosis de ciclosporina, permitieron la curación, como así lo objetivaron las endoscopias y biopsias sucesivas.

Correspondencia:

Dr. J. A. Rivera Bautista

C/ Federico Carlos Sainz de Robles, $17-2^{\circ} \mathrm{H}$ E-28035 Madrid

\section{Bibliografía}

1. Penn I. Horizons in organ transplantation: Malignancy. Surg Clin North Am 1994; 74:1247-1257.

2. Libertiny G, Watson CJE, Gray DWR, Welsh KI, Morris PJ. Rising incidence of post-transplant lymphoproliferative disease in Kidney transplant recipients. Br J Surg 2001; 88:1330-1334.

3. Opelz G, Dohler B. Lymphomas after solid organ transplantation: a collaborative transplant study report. Am J Transplant. 2004; 4:222-230.

4. Harris NL, Swerdlow SH, Frizzera G, Knowles DM. Posttransplant lymphoproliferative disorders. In Jaffe ES, Harris NL, Stein H, Vardiman JW (Eds.): World Health Organization Classification of tumours. Tumours of haematopoietic and lymphoid tissues: pathology and genetics. IARC Press. Lyon 2001

5. Taylor AL, Marcus R, Bradley JA. Post-transplant lymphoproliferative disorders (PTLD) after solid organ transplantation. Crit Rev Oncol Hematol. 2005; 56:155-167.

6. Bakker NA, van Imhoff GW, Verschuuren EA, van Son WJ, Homan van der Heide JJ, Veeger NJ, Kluin PM, Kluin-Nelemans HC. Early onset post-transplant lymphoproliferative disease is associated with allograft localization. Clin Transplant. 2005; 19:327-334.

7. Reyes J, Green M, Bueno J. Epstein Barr virus associated pottransplant lymphoproliferative disease after intestinal transplantation. Transplant Proc. 1996; 28:2768.

8. Holmes RD, Sokol RJ. Epstein-Barr virus and post-transplant lymphoproliferative disease. Pediatr Transplant. 2002; 6:456-464.

9. Molmenti EP, Nagata DE, Roden JS, Squires RH, Molmenti H, Fasola CG, Winick N, Tomlinson G, Lopez MJ,
D’Amico L, Dyer HL, Savino AC, Sanchez EQ, Levy MF, Goldstein RM, Andersen JA, Klintmalm GB. Post-transplant lymphoproliferative syndrome in the pediatric liver transplant population. Am J Transplant. 2001; 1:356-359.

10. Joseph G, Barrer RL, Yuan B, Martin A, Medeiros J, Peiper SC. Posttransplantation Plasma Cell Dyscrasias. Cancer 1994; 74:1959-1964.

11. M Rooney C. New insights into EBV-associated posttransplant lymphoproliferative disease. Lancet. 2003 18; 361:192-193.

12. Leblond V, Sutton L, Dorent R, et al. Lymphoproliferative disorders after organ transplantation: a report of 24 cases observed in a single center. J Clin Oncol 1995; 13:961-968

13. Shitrit D, Shitrit AB, Dickman R, Sahar G, Saute M, Kramer MR. Gastrointestinal involvement of posttransplant lymphoproliferative disorder in lung transplant recipients: report of a case. Dis Colon Rectum. 2005; 48:2144-2147.

14. Sebire NJ, Malone M, Risdon RA, Ramsay AD. EpsteinBarr virus-associated lymphoproliferative disorder presenting as apparently isolated gastrointestinal lesions in childhood. Pediatr Dev Pathol. 2005; 8:88-91.

15. Pimentel RR, van Stolk R. Gastric plasmacytoma: a rare cause of massive gastrointestinal bleeding. Am J Gastroenterol. 1993; 88:1963-1964.

16. Shroff R, Rees L. The post-transplant lymphoproliferative disorder-a literature review. Pediatr Nephrol. 2004 Apr;19(4):369-77.

17. Gleason TH, Hammar SP. Plasmacytoma of the colon: case report with lambda light chain, demonstrated by immunoperoxidase studies. Cancer 1982; 50:130-133.

18. Tsai DE, Aqui NA, Tomaszewski JE, Olthoff KM, Ahya VN, Kotloff RM, Bloom RD, Brozena SC, Hodinka RL, Stadtmauer EA, Schuster SJ, Nasta SD, Porter DL, Luger SM, Klumpp TR. Serum protein electrophoresis abnormalities in adult solid organ transplant patients with posttransplant lymphoproliferative disorder. Clin Transplant. 2005; 19:644-652.

19. Barosi G, Boccadoro M, Cavo M, et al. Management of multiple myeloma and related-disorders: guidelines from the Italian Society of Hematology (SIE), Italian Society of Experimental Hematology (SIES) and Italian Group for Bone Marrow Transplantation (GITMO). Haematologica. 2004; 89:717-741.

20. Green M. Management of Epstein-Barr virus-induced posttransplant lymphoproliferative disease in recipients of solid organ transplantation. Am J Transplant. 2001; 1:103108.

21. Rees L, Thomas A, Amlot PL. Disappearance of an Eptein-Barr virus-positive post-transplant plasmacytoma whit reduction of inmunosuppression. The Lancet, 1998; 352:789.

22. Green M, Reyes J, Webber S, Rowe D. The role of antiviral and immunoglobulin therapy in the prevention of Epstein-Barr virus infection and post-transplant lymphoproliferative disease following solid organ transplantation. Transpl Infect Dis. 2001; 3:97-103. 


\section{J. A. Rivera Bautista y cols.}

23. Stern M, Herrmann R, Rochlitz C, Dirnhofer S, Pless M. A case of post-transplant lymphoproliferative disease presenting as CD20-expressing, Epstein-Barr-virus positive Hodgkin lymphoma. Eur J Haematol. 2005; 74:267-270.

24. Komrokji RS, Oliva JL, Zand M, Felgar R, Abboud CN. Mini-BEAM and autologous hematopoietic stem-cell transplant for treatment of post-transplant lymphoproliferative disorders. Am J Hematol. 2005; 79:211-215.

25. Davis CL. Interferon and cytotoxic chemotherapy for the treatment of post-transplant lymphoproliferative disorder. Transpl Infect Dis. 2001; 3:108-118.

26. Oertel SH, Verschuuren E, Reinke P, Zeidler K, PappVary M, Babel N, Trappe RU, Jonas S, Hummel M, Anagnostopoulos I, Dorken B, Riess HB. Effect of anti-CD 20 antibody rituximab in patients with post-transplant lymphoproliferative disorder (PTLD). Am J Transplant. 2005; 5:2901-2906.
27. Bueno J, Ramil C, Somoza I, Sanchez-Galindo A, Solar A, Arnal F, Alvarez A, Sanchez-Mozo P, Gomez M. Treatment of monomorphic B-cell lymphoma with rituximab after liver transplantation in a child. Pediatr Transplant. 2003;7:153-156.

28. Garcia VD, Bonamigo Filho JL, Neumann J, Fogliatto L, Geiger AM, Garcia CD, Barros V, Keitel E, Bittar AE, Ferrera des Santos A, Roithmann S. Rituximab in association with rapamycin for post-transplant lymphoproliferative disease treatment. Transpl Int. 2003;16:202-206.

29. Davis JE, Moss DJ. Treatment options for post-transplant lymphoproliferative disorder and other Epstein-Barr virus-associated malignancies. Tissue Antigens. 2004 ;63:285-292.

30. Kodama Y, Kawabata K, Yoshida S, Notohara K, Fujimori T, Chiba T. Malt lymphoma simulating an extramedullary plasmacytoma of the stomach. Am J Med. 1999; 107:530532. 